\title{
Uma Abordagem Mista sobre atenção à criança com Necessidades Especiais de Saúde no Brasil
}

\author{
Beatriz Rosana Gonçalves de Oliveira Toso1, Vanessa Rossetto' \\ Cláudia Silveira Viera ${ }^{1}$, Neusa Collet ${ }^{2}$, Mariana Matias Santos ${ }^{2}$, Caren \\ da Silva Bertoldo ${ }^{3}$ e Eliane Tatsch Neves $^{3}$ \\ ${ }^{1}$ Programa de Pós-Graduação Biociências e Saúde, Universidade Estadual do Oeste do \\ Paraná, Brasil | lb.toso@gmail.com | https://orcid.org/0000-0001-7366-077X | \\ vanessa_rossetto@msn.com | https://orcid.org/0000-0003-4902-1534 | \\ clausviera@gmail.com | https://orcid.org/0000-0002-0900-4660 \\ 2 Programa de Pós-Graduação em Enfermagem, Universidade Federal da Paraíba, Brasil | \\ neucollet@gmail.com | https://orcid.org/0000-0002-4795-0279 \\ mari_britomatias@hotmail.com | https://orcid.org/0000-0002-4539-4707 \\ ${ }^{3}$ Programa de Pós-Graduação em Enfermagem, Universidade Federal de Santa Maria, \\ Brasil | carensbertoldo@gmail.com | https://orcid.org/0000-0002-0199-134X | \\ eliane.neves@ufsm.br; https://orcid.org/0000-0002-1559-9533
}

\begin{abstract}
Resumo: Os avanços tecnológicos e a qualificação dos profissionais de saúde, nas últimas décadas, refletiram-se na transição epidemiológica da infância com diminuição da mortalidade infantil, ao mesmo tempo que proporcionaram o crescimento do grupo de crianças que vivem com condições crônicas na infância, nominado crianças com necessidades especiais de saúde. Neste capítulo, descreve-se o cuidado prestado às crianças e adolescentes nos serviços de atenção domiciliar, na perspectiva dos profissionais de saúde e da família, e apresenta-se fluxograma de organização dos serviços para esse público na atenção domiciliar brasileira. Os dados apresentados originaram-se de pesquisa de abordagem quantiqualitativa, do tipo estudo de casos múltiplos. A investigação empregou a triangulação de métodos para obtenção dos dados e foi desenvolvida em duas etapas: a primeira para descrever os serviços prestados e compreender a percepção da família sobre os cuidados recebidos e a segunda para validar fluxograma de organização dos serviços. Foi possível caracterizar os serviços de atenção domiciliar nas distintas realidades, identificando as similaridades e discrepâncias entre as regiões brasileiras. Os familiares apontaram suas perspectivas sobre o cuidado recebido em atenção domiciliar, demonstrando fragilidades e potencialidades do cuidado nos distintos locais. Validou-se um protocolo de fluxo de cuidados a estas crianças, organizando seu trânsito na rede de cuidados.
\end{abstract}

Palavras Chave: Crianças com necessidades especiais de saúde; Serviços de atenção domiciliary; Estudo de casos múltiplos; Triangulação; Protocolo de fluxo de cuidados.

\section{Introdução}

Nas últimas décadas, graças aos avanços da tecnologia e a qualificação dos profissionais de saúde, houve mudanças na transição epidemiológica da infância, com diminuição da mortalidade infantil e neonatal. Entretanto, paralelamente, cresceu o grupo de crianças que vivem com condições crônicas de saúde e com necessidades de cuidados contínuos e complexos, incluindo-se no grupo das crianças com necessidades especiais de saúde (CRIANES)2, denominação publicada em 1998 por McPherson e colaboradores (1998), nos Estados Unidos. Essas crianças demandam cuidados diferenciados, os quais podem abranger uma ou mais das seguintes demandas: reabilitação psicomotora e social, dispositivos e tecnologias, fármacos e cuidados habituais modificados. Assim, o cuidado à saúde dessas crianças deve ser individualizado e contextualizado e ocorrer de forma contínua e dinâmica, compreendendo uma variedade de configurações, impactadas pelas estruturas familiares e de saúde (Brenner et al., 2018). Para atender às necessidades de

\footnotetext{
${ }^{2}$ No Brasil a denominação Children with special healthcare needs foi traduzida livremente como crianças com necessidades especiais de saúde. Porém, nos Estados Unidos da América a palavra children incluiu crianças e adolescentes, sendo que as pesquisas realizadas naquele país com este grupo abrangem a faixa etária de 0 a 17 anos.
} 
saúde desse grupo, impõem-se mudanças na forma de prestar atenção à saúde. Desse modo, cada país desenvolve um modelo de atenção às CRIANES com nível abrangente de cuidados que deve ser acessível e integrado para a criança e sua família e deve ter como base o cuidado domiciliar (Kuo \& Houtrow, 2016).

No Brasil, considera-se a Atenção Domiciliar (AD) uma das respostas do sistema de saúde brasileiro para atender às demandas presentes nas condições crônicas na infância. A portaria $n^{\circ} 825$ de 2016 desenha o modelo de atenção à condição crônica na modalidade atenção domiciliar, cujos objetivos consistem na redução da demanda pela assistência hospitalar, redução da permanência de usuários nas instituições hospitalares, humanização da assistência com a promoção da autonomia dos usuários, e na desinstitucionalização e otimização dos recursos das Redes de Atenção à Saúde - RAS (Brasil, 2016). No entanto, essa portaria organiza algumas premissas para o atendimento em $A D$, mas carece de protocolos de fluxos específicos para atenção à criança nos Serviços de Atenção Domiciliar (SAD). Em vista da recente estruturação das RAS para o atendimento domiciliar, ainda não se tem estudos brasileiros delineando os melhores fluxos para o atendimento das CRIANES, bem como sobre a caracterização desse grupo em atenção domiciliar, configurando-se uma lacuna na produção do conhecimento. A AD brasileira é organizada em três modalidades: AD1, AD2 e AD3. Na AD1, a responsabilidade pela assistência dos usuários é das equipes de atenção primária, tanto na modalidade saúde da família quanto unidades básicas de saúde tradicionais, cujo acompanhamento no domicílio ocorre de acordo com as necessidades dos usuários e disponibilidade das unidades. Na AD2 e AD3, o atendimento ocorre pelo SAD e a modalidade da assistência em $A D$ será definida a partir das necessidades de cuidado do usuário, da demanda de periodicidade das visitas, da intensidade do cuidado multiprofissional e do uso de equipamentos (Brasil, 2016). As crianças e adolescentes atendidos pelo $S A D$, portanto, compreendem a modalidade AD2 e AD3. Nesta última estão aquelas dependentes de tecnologias mais avançadas, como em uso de aparelho de ventilação mecânica, as quais não integraram o estudo, em função de sua condição de saúde, bem como aquelas assistidas pela atenção primária, na modalidade AD1. Desse modo, questiona-se: Quem são as crianças e adolescentes em atenção domiciliar no país? Quais são seus problemas de saúde e suas demandas de cuidado? Qual o fluxo eficaz para a atenção efetiva das CRIANES na RAS?

Este capítulo tem por objetivo descrever o cuidado prestado às crianças e adolescentes com necessidades especiais de saúde nos serviços de atenção domiciliar, na perspectiva dos profissionais de saúde e da família e apresentar protocolo de fluxo de cuidado a crianças com necessidades especiais de saúde na atenção domiciliar, modalidade dois. Apresenta-se um breve histórico sobre o tema, seguido da metodologia adotada em cada etapa da pesquisa e uma síntese dos resultados obtidos.

\section{Tipos de Pesquisa}

Realizou-se uma pesquisa descritiva e exploratória, de abordagem quantiqualitativa, do tipo estudo de casos múltiplos, executada por meio de triangulação de métodos e que se caracteriza como uma pesquisa aplicada, a qual visa encontrar uma solução imediata para um problema existente, com o planejamento de uma mudança para a situação ao final da pesquisa (Polit, Beck, \& Hungler, 2018), executada em duas fases.

A fim de investigar o mesmo fenômeno por meio de duas abordagens de pesquisa, propôsse a realização de estudo de métodos mistos como estratégia metodológica (Yin, 2015), do tipo sequencial explanatório, o qual associa métodos quantitativos e qualitativos de modo sistemático, integrado e crítico, almejando melhor compreensão do fenômeno estudado. Pode ser desenvolvido de forma a que as abordagens quantitativas e qualitativas tenham suas estruturas e procedimentos originais conservados (Creswell, 2014; Sampieri, Collado, \& Lucio, 2013). Na modalidade sequencial, os dados são coletados e analisados em etapas distintas e seriadas, para explorar, ampliar e/ou aperfeiçoar a compreensão sobre a temática em estudo a partir da verificação das informações obtidas na primeira fase (Sampieri et al., 2013). O estudo de caso consiste 
em coletar e analisar informações sobre determinado indivíduo, família, grupo ou comunidade, que tem como objeto o estudo de uma unidade de forma aprofundada, seguindo-se alguns requisitos básicos para sua realização, tais como severidade, objetivação, originalidade e coerência. Sendo um estudo de casos múltiplos, abrange mais de uma realidade, no caso, sete estados brasileiros.

Já a pesquisa qualitativa, para Minayo (2019, p. 25), é importante na construção do conhecimento sobre saúde, visto que os fenômenos referentes a essa área são complexos e "o reconhecimento de sua complexidade dinâmica é requisito indispensável para pensálos cientificamente". Por sua vez, a pesquisa quantitativa busca extrair uma quantificação estatística de tendências do assunto que será explorado e utiliza instrumentos padronizados visando à obtenção de respostas que atendam aos objetivos do levantamento.

A pesquisa que aqui se apresenta foi realizada com caráter descritivo, em que 0 pesquisador observa, conta, descreve e classifica. A abordagem exploratória investiga a natureza do fenômeno e os fatores relacionados (Polit et al., 2018). Essa modalidade permite estudar detalhadamente uma dada situação, no caso, os serviços de atenção domiciliar existentes nos sete estados e seus municípios brasileiros envolvidos, para descrevê-los e evidenciar a existência de protocolos de fluxo de cuidado. A pesquisa foi desenvolvida em duas fases:

\subsection{Fase 1}

Produção de dados quantitativos junto aos serviços de atenção domiciliar, com os profissionais de saúde, para caracterização dos serviços e das crianças em atendimento pelos mesmos, mediante envio de formulário eletrônico para preenchimento online. Paralelamente ocorreu à obtenção de dados qualitativos, por meio de entrevistas presenciais com familiares e/ou cuidadores de CRIANES, ambas com roteiros estruturados.

\subsection{Fase 2}

Validação em sete estados de protocolo originário do estado do Paraná, Brasil, por ocasião da elaboração da dissertação de mestrado de Rossetto (2017), a qual evidenciou a inexistência de fluxogramas organizativos do cuidado a CRIANES, no país.

Os estados são representativos das regiões político administrativas do Brasil. Na região Sul, participam os três estados - Paraná, Santa Catarina e Rio Grande do Sul; na Região Centro-Oeste - Mato Grosso do Sul; na região Sudeste - São Paulo; na região Nordeste Paraíba e Maranhão. Os municípios elencados de cada estado foram todos aqueles que têm SAD registrado e atuante junto ao Ministério da Saúde.

A validação segue metodologia própria de estudos metodológicos para construção e verificação da confiabilidade de instrumentos quanto a face e conteúdo. Foi obtida mediante envio de formulário eletrônico para preenchimento online aos profissionais dos SAD dos estados, seguida de entrevistas presenciais com amostra dos profissionais de saúde, a respeito de sua aplicabilidade.

A pesquisa foi aprovada por comitê de ética em pesquisa com seres humanos, conforme as normativas brasileiras, com parecer número 3.477.776 e CAAE número 17157319.7.1001.0107. 


\subsection{Fases do processo/da pesquisa}

As fases da pesquisa estão sintetizadas no diagrama apresentado na figura 1.

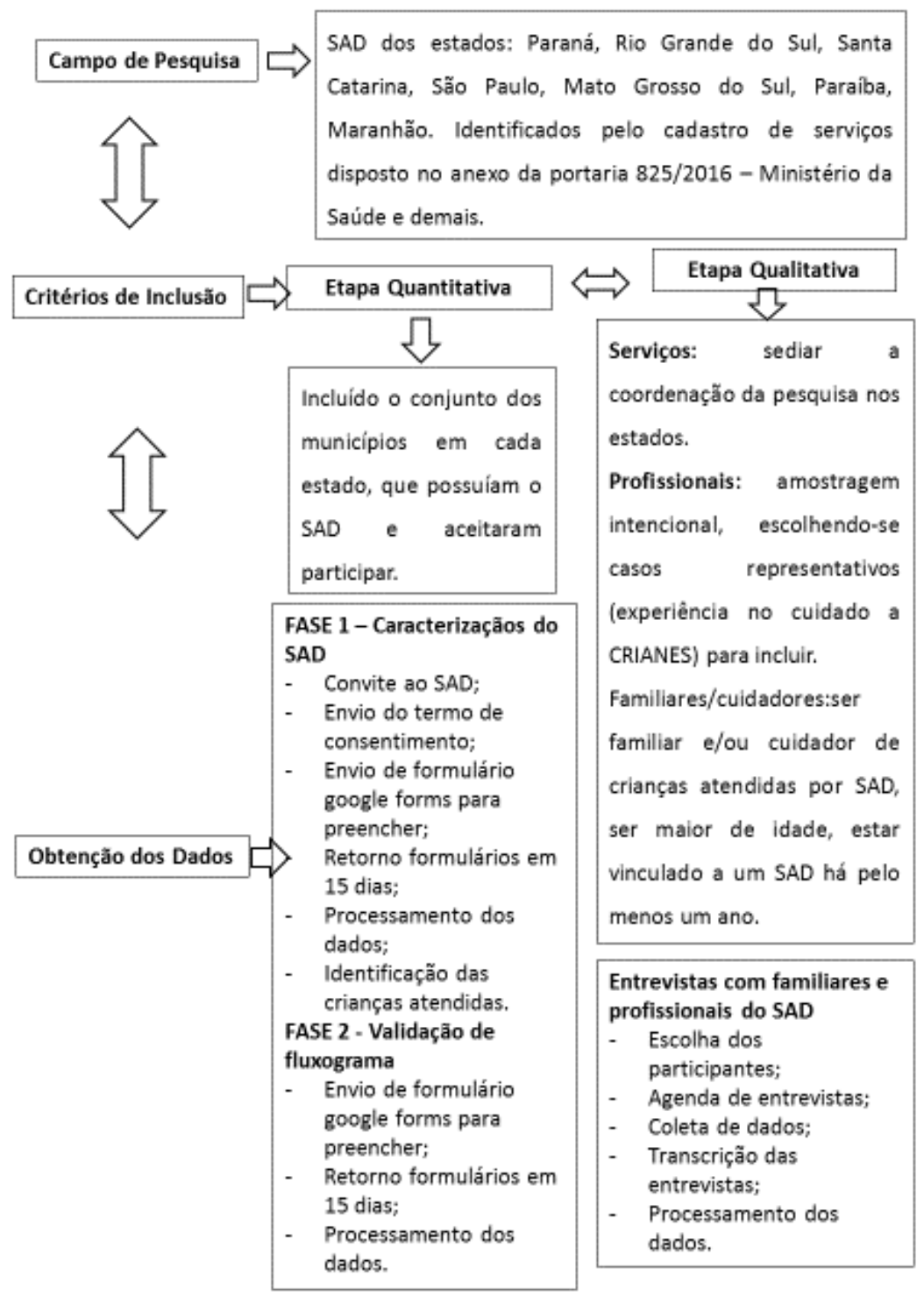

Fig.1. Diagrama de representação das etapas da pesquisa.

Fonte: Elaboração própria.

Na figura 2 apresenta-se o diagrama da fase dois da pesquisa, em relação à validação do protocolo de organização de fluxo, que compreende coleta de dados quantitativos, para validade de face e conteúdo do fluxograma e qualitativos, com entrevistas aos profissionais, os quais integram a mesma fase. 


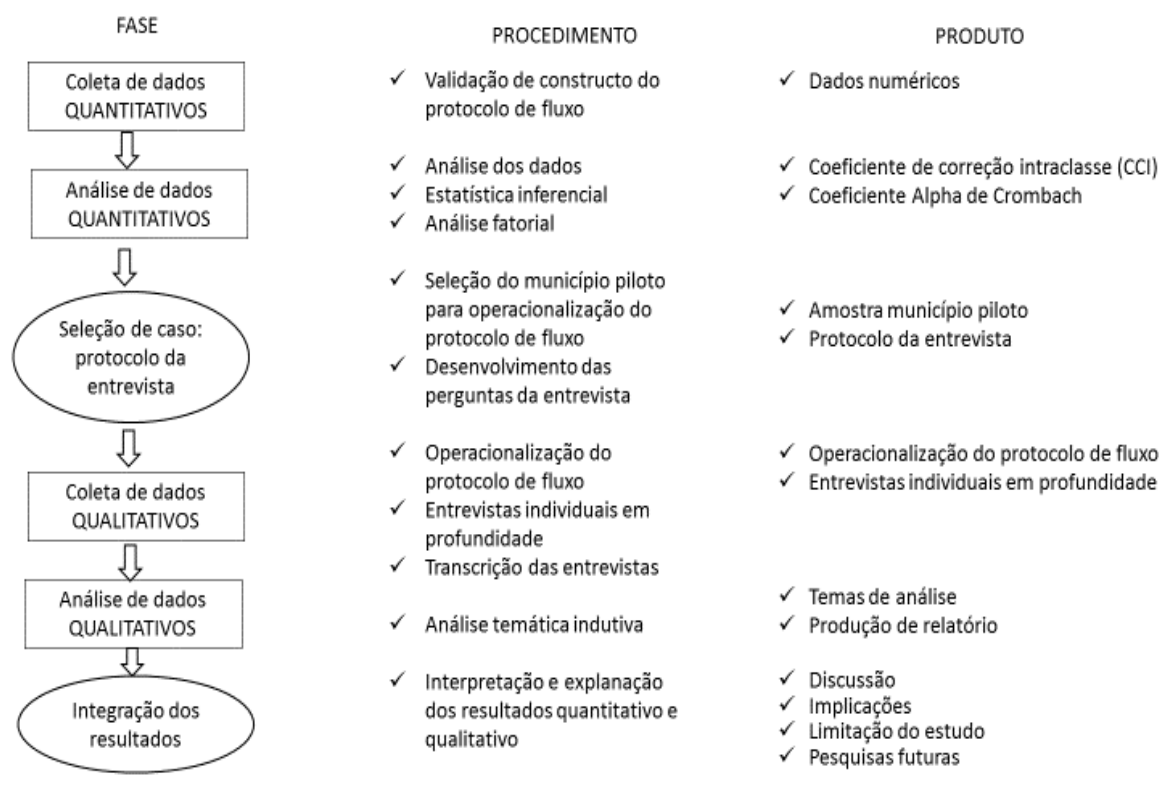

Fig. 2. Diagrama procedural do delineamento misto sequencial explanatório utilizado nas entrevistas com familiares e profissionais. Baseado em Creswell e Clark (2013).

\section{Instrumentos}

Para a coleta de dados quantitativos utilizou-se dois formulários do Google Forms. Como os formulários têm em média 30 páginas, não foi possível sua inclusão na totalidade neste capítulo, apresentando-se abaixo exemplos de questões de cada um deles. As questões iniciais, de caracterização dos serviços e/ou profissionais, são abertas e descritivas, seguidas de questões de múltipla escolha. Para ambos os formulários foi realizado teste piloto. Importante destacar que duas dissertações de mestrado já foram elaboradas e defendidas (Rossetto, 2017; Bertoldo, 2020) e uma tese de doutorado está em finalização, referente à produção desses dados, além dos demais trabalhos científicos em andamento. Portanto, os dados aqui apresentados já foram validados tanto por comitê de ética, quanto por bancas de avaliação de trabalhos científicos. Para a coleta de dados qualitativos utilizou-se roteiro semiestruturado de entrevistas para os familiares e questões norteadoras para os profissionais, conforme excertos de questões, a seguir.

\subsection{Exemplos de questões da entrevista semiestruturada com familiares:}

1) Os profissionais do SAD realizam visita domiciliar para acompanhar o caso da [nome da criança/adolescente]?

2) E, se sim, como você percebe o atendimento que é oferecido para [nome da criança]?

3) $\mathrm{E}$, se não recebe, por qual motivo a criança não recebe atendimento? E onde costuma procurar atendimento?

4) Quando você recebe atendimento de saúde para [nome da criança/adolescente] em casa, quais são os motivos?

- Com qual profissional a(o) [nome da criança/adolescente] é acompanhada?

- Quanto tempo geralmente leva até o atendimento?

- Consegue em seguida agendar consulta de retorno?

- Como você avalia o atendimento recebido por [nome da criança/adolescente]? 
5) Em caso de necessidade de encaminhamentos para especialistas ou outros serviços, como isso ocorre no seu bairro? É você quem agenda ou agendam para você?

6) Você consegue conversar sobre os cuidados que desenvolve com [nome da criança/adolescente] durante o atendimento?

7) Quando você tem dúvidas, quem você procura?

8) Os profissionais disponibilizam tempo para você falar sobre seus problemas ou dúvidas?

Exemplos de questões norteadoras da entrevista com os profissionais:

1) Como você avalia a operacionalização do protocolo de fluxo para o atendimento de CRIANES pelo SAD?

2) Qual a sua percepção sobre a possibilidade de implantação do protocolo de fluxo para atendimento de CRIANES pelo SAD?

3) Você gostaria de expor alguma sugestão ou crítica para melhorar o fluxo das CRIANES na rede? Se sim, explicite-as.

\subsection{Exemplos de questões do formulário de coleta de dados descritivo - funcionamento do SAD.}

1. Identificação do Serviço:

a) Município:

b) Classe profissional a qual você pertence?

c) Qual o número de EMAD? E EMAP?

d) Qual o horário de atendimento do serviço?

e) Quantos profissionais atuam no serviço? Quais?

f) Qual a carga horária de cada profissional?

g) Quem coordena a equipe?

h) Onde está situada a sede do serviço?

i) Descreva as características físicas da unidade.

Tem sede própria?

Quais são os espaços?

Faz atendimento no local ou somente no domicílio?

Quais são as características da estrutura física?

Tem telefone próprio para atendimento aos cuidadores?

Qual é a denominação pela qual o serviço é conhecido no município?

2. Visitas Domiciliares (VD):

Como é feito o deslocamento?

Quais os profissionais da equipe que fazem as VD?

Como se estabelece a rotina de VD (quantas vezes por semana para cada paciente, qual turno?

3. Em relação ao número de pacientes cadastrados, quantos são adultos? Quantos são crianças e adolescentes (0 até 18 anos incompletos)? 
4. Quais os diagnósticos das crianças e adolescentes atendidas (listar todos os diagnósticos de todas as crianças e adolescentes)?

5. Quais as idades das crianças e adolescentes atendidos?

\subsection{Exemplos de questões do instrumento de coleta de dados de validação de fluxograma.}

1. Necessita de acompanhamento em AD2? Marcar apenas uma opção.

( ) $\operatorname{Sim}($ ) Não

Caso tenha respondido "não", dê sua sugestão:

2. NÃO (na figura de decisão do fluxograma): Permanece hospitalizado ou é encaminhado para AD1 ou AD3. Marcar apenas uma opção.

( ) $\operatorname{Sim}($ ) Não

Caso tenha respondido "não", dê sua sugestão:

3. SIM (na figura de decisão do fluxograma): Enfermeiros, médicos, fisioterapeutas e assistentes sociais do hospital enviam ficha de encaminhamento por correio eletrônico para o SAD. Marcar apenas uma opção.

( ) $\operatorname{Sim}($ ) Não

Caso tenha respondido "não", dê sua sugestão:

4. Realizar avaliação de elegibilidade, entre um e sete dias, por profissionais de nível superior da EMAD. Marcar apenas uma opção.

( ) Sim ( ) Não

Caso tenha respondido "não", dê sua sugestão:

5. Há critérios para AD2? Marcar apenas uma opção.

( ) $\operatorname{Sim}($ ) Não

Caso tenha respondido "não", dê sua sugestão:

6. SIM (na figura de decisão do fluxograma): Avaliador de elegibilidade faz a identificação de um cuidador responsável, avaliando sua capacidade de realizar os cuidados necessários e verificando seu aceite para acompanhamento domiciliar. Marcar apenas uma opção.

( ) Sim ( ) Não

Caso tenha respondido "não", dê sua sugestão:

$O$ protocolo de fluxo de cuidados específicos para atenção às crianças com necessidades especiais de saúde na $A D 2$, foi elaborado a partir da identificação de que este é geralmente o grupo mais numeroso na AD. Para tanto, os dados obtidos foram categorizados de acordo com o indicado por Werneck, Farias e Campos (2009) para elaboração de protocolos de organização de serviços e, em seguida, analisados utilizando como instrumento o método SWOT (Strengths, Weaknesses, Opportunities e Threats) (Navarro \& Alencar, 2016). Posteriormente, os pontos elencados foram organizados de acordo com o Plano de Ação 5W2H, para, na última etapa, os resultados serem representados graficamente em formato de fluxograma (Rossetto, 2017; Rossetto, Toso, \& Rodrigues, 2020). 


\section{Participantes}

Os participantes da pesquisa são profissionais dos SAD dos estados participantes e familiares e/ou cuidadores de crianças e adolescentes atendidas por esses serviços. Os serviços foram identificados a partir das portarias de credenciamento, documentos públicos disponíveis junto ao Ministério da Saúde brasileiro. Os profissionais foram contatados a partir do telefone dos serviços e convidados a participar da pesquisa. As famílias, por sua vez, foram identificadas junto aos serviços que realizam atendimento de crianças e convidadas a participar. O número de participantes é variável em cada cenário de pesquisa, pois cada estado tem quantidade diferente de serviços e de profissionais, bem como de crianças em atendimento e como a pesquisa ainda não foi finalizada, pois está em diferentes estágios de andamento em cada estado, esse número ainda não está disponível, apenas do que já foi realizado, em relação aos serviços.

A coleta de dados no estado do Paraná, o primeiro a iniciar as atividades da pesquisa, ocorreu em 2016 e segue em andamento; no estado do Rio Grande do Sul iniciou-se em 2018 e nos demais estados em 2019. A finalização da pesquisa está prevista para 2022.

Os formulários online foram aplicados pelo envio do link do formulário no Google Form para os e-mails informados pelos participantes, os quais responderam e, em tempo real da finalização da resposta, os pesquisadores receberam o instrumento preenchido. Respeitou-se o livre arbítrio na tomada de decisão em querer ou não integrar a pesquisa. Assim, para a obtenção dos dados quantitativos relativos aos serviços, um representante de cada município foi abordado, num total de 132 participantes. A obtenção da amostra dos familiares e/ou cuidadores entrevistados segue os preceitos da metodologia qualitativa e não foi definida a priori.

\subsection{Análise de dados}

\subsubsection{Etapa 1 - Análise Descritiva Quantitativa e Qualitativa}

A análise dos dados quantitativos foi feita por meio de estatística descritiva, a qual é utilizada para descrever e sintetizar os mesmos, permitindo assim, que estes sejam resumidos, organizados e interpretados (Polit et al., 2018). O formulário utilizado produz uma matriz na qual as informações coletadas são agrupadas e organizadas de forma que sua visualização e posterior descrição permitam estabelecer comparações e sínteses, para subsidiar as análises.

Para a análise dos dados qualitativos com familiares e profissionais adotou-se a análise temática indutiva proposta por Braun e Clarke (2006). Trata-se de um tipo de análise de conteúdo de abordagem acessível e flexível, utilizada nas análises de dados qualitativos. A análise temática indutiva é um método para identificar, analisar e relatar temas (padrões) encontrados nos dados, o que permite organizá-los e descrevê-los em detalhe (Braun \& Clarke, 2006). Esta visa analisar e relatar padrões a partir dos dados coletados, os quais são denominados núcleos temáticos e são partes do conjunto de dados, relacionando-se intimamente com a questão de pesquisa. Este tipo de análise encontra-se operacionalmente dividido em seis etapas, de acordo com Braun e Clarke (2006), sendo:

I.Familiaridade com os dados: consiste na transcrição das entrevistas, releituras dos dados e anotação de dados iniciais;

II.Geração de Códigos: Codificação de forma sistemática das características dos dados em comum, agrupamento;

III.Procura de temas: reunião dos dados relevantes para agrupamento e formação de temas potenciais;

IV.Revisão de temas: verificação da relação dos temas com os dados (nível 1) e geração da temática para análise (nível 2); 
V.Definição e Nomenclatura dos temas: análise para refinar a especificidade de cada tema, geração e definição de nomes para cada tema;

VI.Elaboração do relatório: sendo esta a última oportunidade para análise dos extratos.

a. Destaca-se que a ordem não precisa necessariamente ser seguida, o pesquisador tem a liberdade de retornar à primeira etapa, quando julgar necessário.

\subsection{Etapa 2 - Validação de Fluxograma}

O protocolo de fluxo foi avaliado quanto à confiabilidade, propriedade que revela a qualidade do instrumento, e a validade que verifica se este cumpre seu objetivo. A confiabilidade e a validade estabelecem uma relação de dependência em que um instrumento que não é confiável possivelmente não seja válido, enquanto o instrumento pode ser confiável sem ser válido (Polit et al., 2018). Na primeira fase aplicou-se o fluxograma com o público alvo, em todos os serviços de atenção domiciliar dos estados participantes, para a validação de constructo. O protocolo foi enviado aos serviços existentes para avaliação de face e conteúdo e, posteriormente, para aplicação. Os dados obtidos foram submetidos à análise fatorial com método de rotação Varimax para rotação dos fatores pela normalização de Kaiser. A confiabilidade do instrumento foi avaliada pelo coeficiente de correlação intraclasse (CCl), visando avaliar sua reprodutibilidade, em que valores menores que 0,4 indicam pobre reprodutibilidade, valores entre 0,4 e 0,75 revelam satisfatória reprodutibilidade, e valores acima ou iguais a 0,75 , excelente reprodutibilidade (Cortés-Reyes, Rubio-Romero, \& Gaitán-Duarte, 2010). A avaliação da consistência interna dos dados foi realizada com a aplicação do coeficiente de Alpha de Cronbach, pois de acordo com Hair et al. (2009) os valores de Alpha de Cronbach acima de 0,8 são considerados adequados, embora valores de 0,7 sejam aceitáveis. A validade do instrumento foi verificada por meio da validação de construto, que possibilita uma construção representativa do verdadeiro significado teórico de um conceito (Martins, 2006). Os valores obtidos com o teste e sua validação clínica foram submetidos à análise fatorial com o método de rotação Varimax para rotação dos fatores pela normalização de Kaiser.

\section{Diretrizes para outros estudos}

A experiência relatada pode ser modificada, com outros olhares para as mesmas questões, por exemplo:

- Conduzir pesquisas multicêntricas quantiqualitativas e/ou de métodos mistos possibilitando a produção de conhecimento singular de cada um dos métodos e técnicas, bem como das particularidades loco regionais;

- Adoção do consumo dos resultados de pesquisa quantiqualitativa para a prática baseada em evidências;

- Descrição dos fatores que indicam potencialidades e fragilidades em desenhos de pesquisa quantiqualitativa e a transferência de conhecimento para a prática.

- Como potencialidades do método adotado evidencia-se a ampla possibilidade de obtenção de dados das mais diversas naturezas e possibilidades de análise. A utilização de métodos mistos na modalidade adotada propicia ainda a geração de uma nova e mais abrangente conjunção de dados, com novos olhares e distintos resultados do que somente aqueles produzidos em cada uma das etapas;

- Por sua vez, essa mesma amplitude gera fragilidades, ainda mais em estudo multicêntrico, com sete distintos locais de coleta de dados, com tempos diferentes entre si, num país de distâncias geográficas continentais, uma vez que muitas pesquisas derivam dessa maior, com dissertações de mestrado sendo produzidas, teses de doutorado, iniciação científica, o que é um desafio na gestão da condução do grupo de pesquisa; 
- Ressalta-se que a comunicação virtual foi e tem sido um excelente mecanismo de trabalho entre o grupo, possibilitando reuniões de discussão e esclarecimentos de dúvidas, bem como de discussão dos resultados da pesquisa.

\section{Aplicabilidade em outros contextos}

O tema proposto pode ser aplicado em qualquer realidade de atenção primária, desde que consideradas as diferenças loco regionais e culturais. Debater a forma de organização do cuidado para CRIANES desempenhada no Brasil, demonstrando como é o fluxo organizativo dos cuidados, com exemplos de resultados dos estados brasileiros onde é desenvolvida, potencializa a replicação em outros países com atenção primária organizada, principalmente aqueles com saúde da família, mas não somente. Isso decorre da alteração nas necessidades especiais de cuidados das crianças e adolescentes com condições crônicas, cujo perfil tem mudado ano a ano, acompanhando o avanço das tecnologias em saúde e o aprimoramento dos profissionais de saúde. A promoção de um trabalho integrado entre o que a academia produz e a práxis consome, pode garantir cuidados de qualidade, seguros e acessíveis, mas acima de tudo é uma questão de equidade, universalidade e integralidade para o cuidado às CRIANES paralelamente ao desenvolvimento de um sistema de saúde efetivo.

Neste estudo, desenvolveu-se uma tecnologia de cuidado por meio da elaboração de fluxograma organizativo de cuidados às CRIANES. Isso mostra o compromisso dos pesquisadores com a identificação de prioridades para a qualidade e segurança dos cuidados de saúde às crianças, em rede multicêntrica para o desenvolvimento do conhecimento e sua aplicação prática, em linha de investigação sinérgica com os objetivos dos serviços, possibilitando a introdução da evidência científica produzida por meio da pesquisa, por meio de translação do conhecimento e consumo de resultados de pesquisa no cotidiano da prática assistencial.

\section{Conclusões}

A partir dos métodos utilizados, os quais se mostraram adequados ao cumprimento dos objetivos do estudo, foi possível caracterizar os serviços de atenção domiciliar nas distintas realidades que compõem o estudo multicêntrico, identificando as similaridades e discrepâncias entre as regiões brasileiras, utilizando-se a coleta de dados quantitativos com formulário online e análise descritiva. Para os familiares entrevistados, evidenciado pelo uso da entrevista e a análise qualitativa, estes apontaram suas perspectivas sobre o cuidado recebido em atenção domiciliar, demonstrando fragilidades e potencialidades do cuidado nos distintos locais. Paralelamente, foi validada uma proposta de protocolo de fluxo de cuidados a estas crianças, uma vez que é necessário que o processo de transferência do cuidado para o domicílio esteja claramente organizado, demonstrando que a coleta de dados por meio de formulário online, produzindo resultados em tempo real, é um método adequado de coleta de dados e auxilia na interpretação descritiva destes. $O$ prazo para avaliação da elegibilidade deve estar estabelecido, considerando o tempo necessário para identificação do cuidador, a avaliação de sua capacidade de realizar os procedimentos necessários no domicílio, o aceite do cuidador para o acompanhamento domiciliar, a avaliação do domicílio, se indicado, e a organização da remoção do paciente. Assim, um protocolo organizativo do fluxo que foi desenvolvido em pesquisa realizada no estado do Paraná, Brasil, no âmbito da $A D$, que, diferentemente dos protocolos clínicos, caracteriza-se como protocolo de organização dos serviços, a partir de então, vem sendo testado nos diferentes Estados e regiões brasileiras. Ressalta-se que, dentre os profissionais de saúde que compõem o SAD, o enfermeiro, enquanto integrante das equipes de saúde e gestor do cuidado, tem grande potencial para o atendimento das condições crônicas na AD. Dessa maneira, no cuidado domiciliar às crianças, o enfermeiro precisa estar atento para acolher às demandas das CRIANES e das famílias e sistematizar a assistência que será oferecida. Acredita-se na potencialidade de replicação do estudo para outras realidades. Por fim, utilizar métodos mistos nesta pesquisa possibilitou 
compreender de forma mais abrangente o objeto do estudo, fase ainda em andamento, com potencial de produzir mais e novos resultados que agregarão conteúdo ao conhecimento disponível.

\section{Referências}

Bertoldo, C.S. (2020). Serviço de atenção domiciliar às crianças com necessidades especiais de saúde no sul do Brasil (Dissertação de Mestrado). Universidade Federal de Santa Maria UFSM. Santa Maria, RS.

Brasil. (2016). Ministério da Saúde. Portaria $n^{\circ} 825$ de 25 de abril de 2016. Brasília: MS.

Braun, V., \& Clarke, V. (2006). Using thematic analysis in psychology. Qualitative Research in Psychology, 3(77), 77-101. DOI: 10.1191/1478088706qp063oa

Brenner, M., Kidston, C., Hilliard, C., Coyne, I., Eustace-Cook, J., Doyle, C., Begley, T., \& Barrett, M. J. (2018). Children's complex care needs: a systematic concept analysis of multidisciplinary language. European Journal of Pediatrics, 177(11), 1641-1652. https://doi.org/10.1007/s00431-018-3216-9

Creswell, J.W. (2014). Research design: qualitative, quantitative, and mixed methods approaches (4. ${ }^{a}$ Ed.). SAGE Publications.

Creswell, J.W, \& Clark, V.L.P. (2013). Pesquisa de métodos mistos. 2. Ed., Porto Alegre: Penso.

Cortes-Reyes, E., Rubio-Romero, J.A., \& Gaitan-Duarte, H. (2010). Statistical methods for evaluating diagnostic test agreement and reproducibility. Rev Colomb Obstet Ginecol, 61(3), 247-255.

Hair, J.F. (2009). Análise multivariada de dados (6. ${ }^{a}$ ed.), Porto Alegre: Bookman.

Lakatos, E.M., \& Marconi, M.A. (2011). Metodologia científica. São Paulo: Atlas.

Kuo, D.Z., \& Houtrow, A.J. (2016). Recognition and Management of Medical Complexity. Pediatrics, 138(6), e20163021. https://doi.org/10.1542/peds.2016-3021

Martins, G.A. (2006). Sobre confiabilidade e validade. Revista Brasileira de Gestão de Negócios, 8(20), 1-12.

McPherson, M., Arango, P., Fox, H., Lauver, C., McManus, M., Newacheck, P. W., Perrin, J. M., Shonkoff, J. P., \& Strickland, B. (1998). A New Definition of Children With Special Health Care Needs. Pediatrics, 102(1), 137-139. https://doi.org/10.1542/peds.102.1.137/

Minayo, M.C.S. (Org.). (2019). Pesquisa Social: teoria, método e criatividade. Petrópolis, RJ: Vozes.

Navarro, F.A.R., \& Alencar, C. (2016). Análise dos elementos estratégicos em processos de internacionalização: empresas brasileiras de real estate visando ao mercado residencial da Costa Rica. Ambiente Construído, 16(1), 285-299. https://doi.org/10.1590/s167886212016000100074

Polit, B., Beck, C.T., \& Hungler, D. (2018). Fundamentos de pesquisa em enfermagem. Porto Alegre: Artmed.

Werneck, M.A.F., Faria, H.P., \& Campos, K.F.C. (2009). Protocolo de cuidados à saúde e de organização do serviço. Belo Horizonte: Nescon/UFMG, Coopmed.

Yin, R.K. (2015). Estudo de caso: planejamento e métodos (5. ${ }^{a}$ ed). Porto Alegre: Bookman.

Rossetto, V. (2017). Protocolo de Fluxo de Cuidado Domiciliar para a Criança com Necessidades Especiais de Saúde no Paraná (Dissertação de Mestrado). Universidade Estadual do Oeste do Paraná. Cascavel, UNIOESTE.

Rossetto, V., Toso, B.R.G.O., \& Rodrigues, R.M. (2020). Fluxograma organizativo de atenção domiciliar às crianças com necessidades especiais de saúde. Revista Brasileira de Enfermagem, 73(sup.4), e20190310. http://dx.doi.org/10.1590/0034-7167-2019-0310

Sampieri, R.H., Collado, C.F., \& Lucio, M.P.B. (2013). Metodologia de pesquisa (5. ${ }^{a}$ Ed). Porto Alegre: Penso. 


\section{Nota dos autores}

Todas as autoras são pesquisadoras que integram a pesquisa desde sua concepção, coleta de dados e análise de dados e contribuíram igualmente na redação do capítulo.

\section{Notas biográficas}

Beatriz Rosana Gonçalves de Oliveira Toso. Enfermeira, doutora em Ciências, pós-doutora na atenção primária, professora associada da Universidade Estadual do Oeste do Paraná, do Curso de Graduação em Enfermagem, na área de Saúde da Criança e do Adolescente e do programa de pósgraduação Biociências e Saúde. Membro do Grupo de Pesquisa em Enfermagem Materno-Infantil GPEMI. Vice-presidente da Sociedade Brasileira de Enfermeiros Pediatras - SOBEP. Coordenadora do PROENF/SCA - Saúde da Criança e do Adolescente. Membro da Red Internacional de Enfermería en Salud Infantil (RED-ENSI).

Vanessa Rossetto. Enfermeira, Mestre em Biociências e Saúde, Doutoranda do Programa de PósGraduação em Biociências e Saúde da Universidade Estadual do Oeste do Paraná. Enfermeira do Programa de Atenção Domiciliar do município de Cascavel - PR. Professora do Curso de Enfermagem da Universidade Paranaense.

Cláudia Silveira Viera. Enfermeira, doutora em Enfermagem em Saúde Pública, pós-doutora em seguimento do recém-nascido de risco na atenção primária, professora associada da Universidade Estadual do Oeste do Paraná, do Curso de Graduação em Enfermagem, na área de Saúde da Criança e do Adolescente e do programa de pós-graduação Biociências e Saúde. Membro do Grupo de Pesquisa em Enfermagem Materno-Infantil - GPEMI. Representante do Brasil no Council of International Neonatal Nurses - COINN. Membro da Red Internacional de Enfermería en Salud Infantil (RED-ENSI).

Neusa Collet. Enfermeira, doutora em Enfermagem, professora Associada IV do Departamento de Enfermagem de Saúde Coletiva da Universidade Federal da Paraíba. Docente do Programa de PósGraduação em Enfermagem (Mestrado e Doutorado) e do Curso de Mestrado Profissional em Saúde da Família da Rede Nordeste de Formação em Saúde da Família (RENASF-FIOCRUZ), Nucleadora UFPB. Pesquisador 2 do CNPq. Líder do Grupo de Estudos e Pesquisa em Saúde da Criança e do Adolescente (GEPSCA). Membro da Sociedade Brasileira de Enfermeiros Pediatras e Neonatal (SOBEP). Membro da Red Internacional de Enfermería en Salud Infantil (RED-ENSI).

Mariana Matias Santos. Enfermeira, Especialista em Pediatria e Neonatologia. Mestra em Enfermagem pela Universidade Federal da Bahia. Doutoranda em Enfermagem na Universidade Federal da Paraíba.

Caren da Silva Bertoldo. Enfermeira, Mestre em Enfermagem pelo PPGEnf-UFSM (Bolsista CAPES no período de abril de 2018 a março de 2020). Doutoranda em Enfermagem pelo programa de Pós-graduação em Enfermagem da Universidade Federal de Santa Maria (PPGEnf-UFSM), linha de pesquisa Cuidado e Educação em Enfermagem e Saúde. Membro egresso do Programa de Educação Tutorial (PET) da Universidade Federal de Santa Maria. Membro do Grupo de Pesquisa Saúde do Neonato, Criança, Adolescente e Família - CRIANDO/UFSM.

Eliane Tatsch Neves. Enfermeira pediatra. Doutora em Enfermagem (EEAN/UFRJ) com Doutorado Sanduíche na McGill University (Montreal/Canadá), pós-doutora em Enfermagem em Saúde Pública (EERP/USP), professora Associada do Departamento de Enfermagem da Universidade Federal de Santa Maria do Curso de Graduação em Enfermagem, na área de Saúde da Criança e do Adolescente. Docente do Programa de Pós-Graduação em Enfermagem (Mestrado e Doutorado). Bolsista de Produtividade em Pesquisa do CNPq - PQ2. Líder do Grupo de Pesquisa Saúde do Neonato, Criança, Adolescente e Família - (CRIANDO/UFSM). Membro da Comissão Permanente de Titulação da Sociedade Brasileira de Enfermeiros Pediatras e Neonatal (SOBEP). Membro da Red Internacional de Enfermería en Salud Infantil (RED-ENSI). 